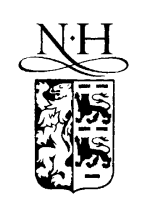

ELSEVIER

\title{
Low-temperature investigation of paramagnetic centres in tetrahedral amorphous carbon deposited by S-bend FCVA
}

\author{
G. Fanchini ${ }^{\text {a,* }}$, A. Tagliaferro ${ }^{\text {a }}$, D. Dasgupta ${ }^{\text {a,1 }}$, E. Laurenti ${ }^{\text {b }}$, A.C. Ferrari ${ }^{\text {c }}$, \\ K.B.K. Teo ${ }^{\mathrm{c}}$, W.I. Milne ${ }^{\mathrm{c}}$, J. Robertson ${ }^{\mathrm{c}}$ \\ a Dip. Fisica and Unità INFM, Politecnico di Torino, Corso Duca degli Abruzzi 24, I-10129 Torino, Italy \\ ${ }^{\mathrm{b}}$ Dip. Chimica IFM, Università degli Studi di Torino, I-10125 Torino, Italy \\ ${ }^{\mathrm{c}}$ Engineering Department, University of Cambridge, Cambridge CB2 1PZ, UK
}

\begin{abstract}
The electron-spin-resonance (ESR) features of tetrahedral amorphous carbon (ta-C) samples deposited by S-bend filtered cathodic vacuum arc (FCVA) are investigated in a wide temperature range (4.3-295 K) before and after postdeposition annealing at $500{ }^{\circ} \mathrm{C}$. Special attention is given to the temperature dependence of the linewidths. While the sp ${ }^{3}$ content does not change with annealing, the temperature trend of the linewidth becomes much smoother for postdeposition annealed samples. The results are discussed in terms of different possible mechanisms of motional linenarrowing and line-narrowing due to hopping motion. (c) 2002 Elsevier Science B.V. All rights reserved.
\end{abstract}

PACS: 81.05.uw; 76.30.-v; 66.35.+a

\section{Introduction}

In amorphous carbons [1], the nature of the defects is still unclear, although, at least for a-C:H, some explanation was recently attempted [2] in terms of defects of (micro-)crystalline graphite. This explanation, however, cannot account for the nature of paramagnetic centres in tetrahedral amorphous carbon (ta-C), since such material does

\footnotetext{
${ }^{*}$ Corresponding author. Tel.: +39-011 564 7381; fax: +390115647399 .

E-mail address: fanchini@polito.it (G. Fanchini).

${ }^{1}$ Permanent address: Physics Department, North Bengal University, Siliguri, India
}

not contain neither $\mathrm{sp}^{2}$ aromatic islands nor dangling bonds but, more likely, $\mathrm{sp}^{2}$ sites clustered in chains and/or isolated $\mathrm{sp}^{2}$ sites [1,3]. On the other hand, besides the nature of defects, it is crucial to understand their behaviour in the framework of the transport properties. Such behaviour can be investigated by electron-spin-resonance (ESR) spectroscopy.

Despite amorphous carbons [1-3] and amorphous silicon are so different, a common point is that, in each of these two classes of materials, the transport of charge and the transport of the spin excitation present some analogies [4,5]. Since the ESR linewidths are related with the spin relaxation times, the temperature trends of the ESR linewidth are powerful tools to investigate the mobility processes of unpaired electrons [4,5]. Indeed, if the 
signals are hopping- or motionally narrowed, the linewidths decrease with temperature. Following this approach, it is clear that, in order to get a deeper understanding of the spin relaxation times, one needs data acquired at various temperatures. Here we present a temperature investigation of the spin relaxation processes in ta-C. The nature of the spin-spin interaction will be discussed.

\section{Experimental}

Ta-C films were simultaneously deposited on quartz and Si substrates using a filtered cathodic vacuum arc (FCVA) system with an off-plane double bend (S-bend) magnetic filter. An optimal filtering of the graphitic macroparticles is obtained with this system. Thus, ta-C films homogeneous at the nano scale are consistently deposited [6]. Our samples have $\sim 85 \% \mathrm{sp}^{3}$ as determined by electron energy loss spectroscopy (EELS) [6].

ESR measurements were performed in the Xband $\left(\omega_{\mu W} \sim 10 \mathrm{GHz}\right)$ with a Bruker ESP300E spectrometer. The spin densities and the $g$-factors were obtained at room temperature by comparison of the film signal with the reference signal generated by water solutions of TEMPO at known dilution. The silicon substrates were then etched and ta-C powder collected. The etching process is the same used to prepare the EELS specimens. Room temperature measurements were performed in order to check the consistency of the results obtained for the powder and for films deposited on quartz and silicon substrates. Low-temperature measurements were performed on the powder under static vacuum $\left(10^{-3}\right.$ mbar $)$ at liquid nitrogen $(77 \mathrm{~K})$ and, down to $4.3 \mathrm{~K}$, in a liquid-helium cryostat. At each temperature, microwave powers between 1 to $160 \mathrm{~mW}$ were used in order to check the saturation behaviour of the signal and to estimate (under the assumptions of homogeneous system, and $\tau_{1} \ll \tau_{2}$ $[7,8])$ the spin-lattice $\left(\tau_{1}\right)$ and spin-spin $\left(\tau_{2}\right)$ relaxation times. The powder and one of the quartzdeposited films were then annealed at $500{ }^{\circ} \mathrm{C}$ in vacuum $\left(3-5 \times 10^{-5} \mathrm{mbar}\right)$ in a tubular oven. The ESR measurements were then repeated. The EELS measurements showed that no change in $\mathrm{sp}^{3}$ content occurred after this annealing procedure.

\section{Results}

By considering the series of ESR measurements recorded at different temperatures, we note that the signal linewidth increases as the temperature decreases (Fig. 1). In the as-deposited sample and above $T_{0}=15 \mathrm{~K}$, the signal is Lorentzian (Fig. 2). However, for $T<T_{0}$ a transition occurs, the signal line-shape becomes a Gaussian and Lorentzian mixture and there is a steep increase of the signal linewidth. After annealing the $\mathrm{sp}^{3}$ content remains unchanged, but a spin density reduction from $7.5( \pm 1.5)$ to $2.5( \pm 0.5) \times 10^{20} \mathrm{~cm}^{-3}$ occurs and a less marked decrease of the ESR linewidth with temperature is found (Fig. 1). The transition at $T_{0}=15 \mathrm{~K}$ disappears and the signal has the same (Lorentzian) line-shape from 295 to $4.3 \mathrm{~K}$. The integrated area of the spectra always increases with the Curie law [7]. The $g$ factor $(2.0024 \pm 0.0002)$ is same before and after annealing.

The spectra recorded at different micro wave power show that the spin-lattice relaxation times $\tau_{1}$ are very fast, both in the as-deposited and the annealed sample. At room temperature and down to $15 \mathrm{~K}$, no saturation of the signal is detected up to $160 \mathrm{~mW}$. This means that $\tau_{1}$ can be estimated to be $<5 \times 10^{-8}$ s [7]. Only below $15 \mathrm{~K}$ a weak saturation can be observed. At $6 \mathrm{~K}$, the spin-lattice relaxation times are estimated to be $\tau_{1}=1 \times 10^{-7} \mathrm{~s}$

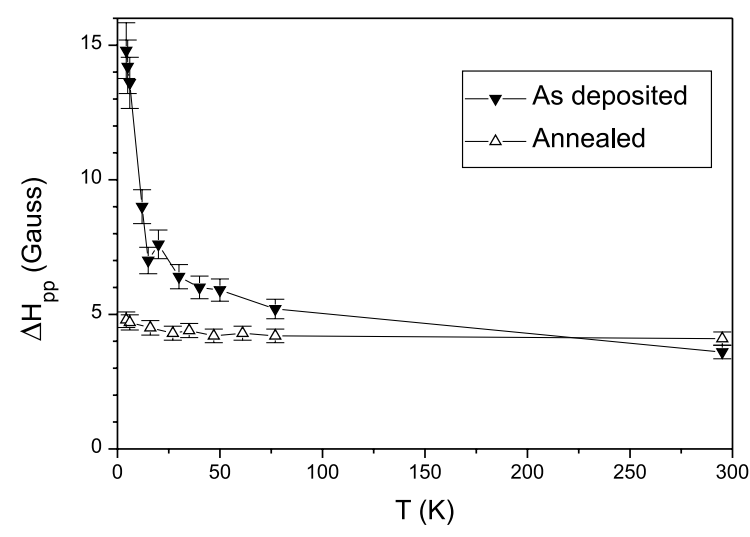

Fig. 1. ESR linewidths of two ta-C samples (as-deposited and post-deposition annealed to $500{ }^{\circ} \mathrm{C}$ ) as a function of the measurement temperature. The sharp trend of the as-deposited samples becomes much smoother in the post-deposition annealed ones. 


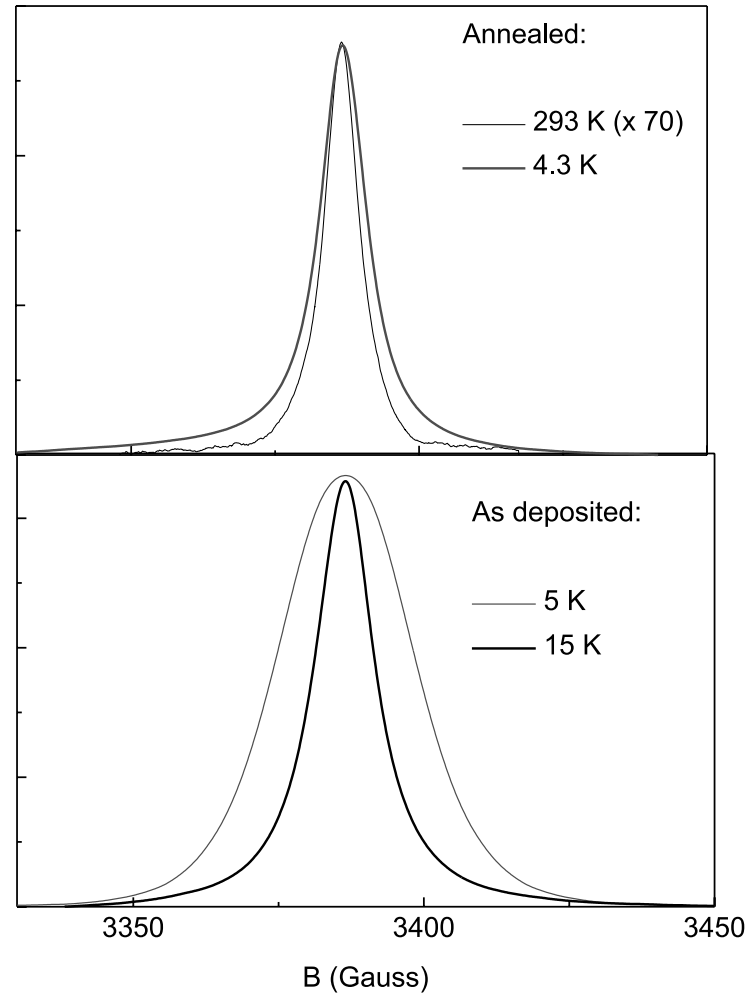

Fig. 2. Integrated ESR signals of the ta-C samples of Fig. 1. The change in lineshape of the as-deposited sample when measured at $5 \mathrm{~K}$ has no counterpart for the post-deposition annealed one.

and $\tau_{1}=8 \times 10^{-8} \mathrm{~s}$ for the as-deposited and annealed samples, respectively. The spin-lattice relaxation times $\tau_{1}$ are longer than $5 \times 10^{-8} \mathrm{~s}$ only at $T \sim T_{0}$ (i.e. when the as-grown sample approaches the sharp increase of the linewidth) so that, at such temperature, $\tau_{1} \sim \tau_{2} \sim 1 \times 10^{-8}$. This means that the spin-lattice relaxation times we can calculate by the saturation method (i.e. under the assumption $\tau_{1} \gg \tau_{2}$ see above) are meaningful in their actual values only at $T<T_{0}$. At the opposite, for $T>T_{0}$ the primary channel of immediate spin-to-spin energy transfer competes with a 'secondary' channel involving a 'mediated' energy transfer: first, from an excited spin to the lattice; second, from the lattice to another spin (i.e. a strong hopping mechanism). As $\tau_{1}$ decreases much faster than $\tau_{2}$ with temperature [7], the primary channel will be readily short-circuited by the other as the tem-

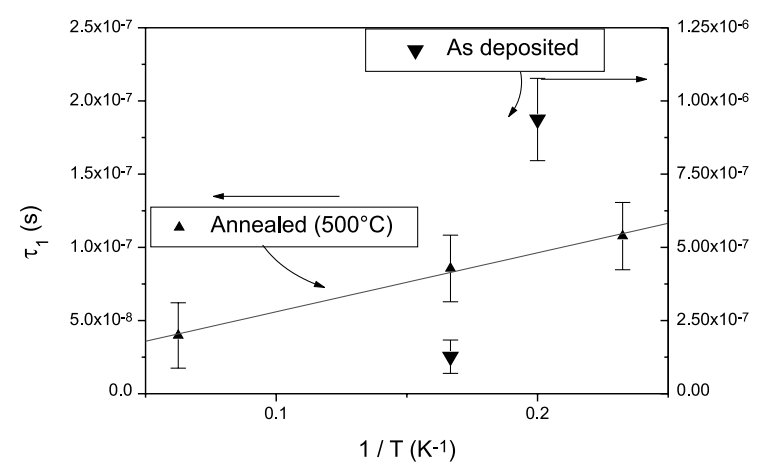

Fig. 3. Spin-lattice relaxation time $\tau_{1}$ at different temperatures. The data scale with the inverse of the temperature, suggesting the relaxation to occur by direct processes.

perature increases. Only below $T_{0}$ (i.e. at the lowest $T$ ) due to the increase of $\tau_{1}$ (now measurable with saturation methods) the non-mediated (primary) spin-spin channel comes into play. According to our data (Fig. 3) $\tau_{1}$ decreases with temperature as $T^{-1}$. This means [7] that the spin excitation is absorbed by direct processes exciting long wavelength lattice vibrations, resonant with the Larmor frequency. Since the spin-lattice relaxation does not depend on the spin-spin relaxation, a similar trend $\tau_{1}(T) \propto T^{-1}$ and similar processes probably occur also at $T>T_{0}$. This is also supported by the results obtained in a-C(:H) where $\tau_{1}$ can be measured by the saturation method up to room temperature and shown to decrease as $T^{-n}(1<n<2)$ [4]. The simultaneous occurrence of $\tau_{1} \sim \tau_{2}$ and direct spin-lattice processes (exciting lattice vibrations at frequencies $\omega_{0}$ comparable to the Larmor frequency $\omega_{\mu \mathrm{W}}$ ) show that the role of the lattice is crucial in determining the temperature dependence of the linewidth.

Finally, from the spin densities, the nonrandomised distances between point-like spins are $2 R_{\mathrm{S}}=14$ and $20 \AA$, respectively, before and after sample annealing. They correspond to randomised [13] spin-spin distances of $2 R=(3 / 4) 2 R_{\mathrm{S}}=10$ and $15 \AA$. Both distances are quite larger than the $\mathrm{sp}^{2}$ cluster sizes in ta-C (3-5 $\AA$, if we hypothesise the $\mathrm{sp}^{2}$ sites to be in short chains and we assume a $\mathrm{C}=\mathrm{C}$ pair to be $\approx 1.4 \AA$ long [1]). This means that, at $0 \mathrm{~K}$, the unpaired electrons are not overlapped and the linewidths are not exchange-narrowed but 
the distances are not so large to consider the paramagnetic centres to be diluted.

\section{Discussion}

In ta-C, the only active nucleus present $\left({ }^{13} \mathrm{C}\right)$ has a low abundance $(\sim 1 \%)$, ruling out the hypothesis of unresolved hyperfine broadening. In all amorphous carbons, the high field data (Q- and W-band) have shown that no or little change in the linewidth occurs by changing the microwave frequency $\omega_{\mu W}$, ruling out the line broadening due to a spread of the $g$ factors [2,9]. This led to assume [8] the main source of line broadening to be the dipole-dipole interaction. In an homogeneous system with $N_{\mathrm{s}}$ spins per $\mathrm{cm}^{3}$ and $g \approx 2$, the dipolar broadening of the signal (in Gauss) is found to follow the law $\Delta H_{\text {Dip }} \approx 8 \times 10^{-20} N_{\text {s }}$ [8], (i.e. $\Delta H_{\text {Dip }}=60 \mathrm{G}$ before and $\Delta H_{\text {Dip }}=20 \mathrm{G}$ after annealing). At each considered temperature, the purely dipolar linewidths are higher than the real ones: $\Delta H_{\text {Dip }}>\Delta H_{\mathrm{pp}}$, indicating that a temperature dependent line-narrowing mechanism comes into play. Three different line-narrowing models will be discussed in the following sections.

\subsection{Floating bonds}

In poly-Si(:H) films Nickel and Schiff (NS) [10] assume the linewidth to be motionally narrowed due to the migration of the dangling bonds in the lattice, but no temperature effect was found by the authors in amorphous-Si:H. They estimated the linewidth as

$\Delta H_{\mathrm{pp}}=\Delta H_{\mathrm{dip}} /\left[1+\tau_{1}(T) / \tau_{\mathrm{C}}(T)\right]$.

However, assuming a diffusion rate $1 / \tau_{\mathrm{C}}=$ $\omega_{0} \exp \left(-E_{\mathrm{B}} / k_{\mathrm{b}} T\right)$ (as it would be assumed for floating bonds in the lattice) Eq. (1) provides curved-down lines [10], not fitting our trends, that are curved-up (Fig. 2). Actually, Eq. (1) is not valid when $\tau_{1} \sim \tau_{2}$. Beside these evidences, the use of the NS model on ta-C is unphysical. The paramagnetic centres are very different with respect to the poly-Si(:H) ones: they are not diluted dangling bonds but, more probably, $\mathrm{sp}^{2}$ sites or clusters $[1,3]$ which migration, if occurring, would require the break and reconstruction of more (and more rigid) bonds. In our knowledge, there is no model supporting the migration of $\mathrm{sp}^{2}$ sites in ta-C. Moreover in poly-Si(:H) the effect was at $T>$ $300 \mathrm{~K}$ while our linewidths sharply change at low $T$.

\subsection{Non-floating bonds and variable range hopping}

Another explanation (originally proposed for glow-discharge deposited a-Si [11]) involves the line-narrowing hopping motion of the electrons in the lattice, with the ionisation of part of the isolated $\mathrm{sp}^{2}$ sites or odd-fold clusters responsible to the ESR signal, but without any bond breaking or reforming. In the presence of pure dipolar broadening the linewidth can be written [12]

$$
\begin{aligned}
& \Delta H_{\mathrm{pp}}=\frac{1}{\gamma \tau_{2}} \approx \frac{\Delta H_{\mathrm{dip}}^{2} \tau_{\mathrm{C}}(T)}{\gamma} \text { for } \omega_{\mu W} \tau_{\mathrm{C}} \gg 1 ; \\
& \Delta H_{\mathrm{pp}}=\frac{1}{\gamma \tau_{2}} \approx \frac{2 \Delta H_{\mathrm{dip}}^{2} \tau_{\mathrm{C}}(T)}{\gamma} \text { for } \omega_{\mu \mathrm{w}} \tau_{\mathrm{C}} \ll 1 .
\end{aligned}
$$

Eq. (2) represents two 'extreme' behaviours, the former corresponding to $\tau_{1} \ll \tau_{2}$, the latter (valid for $\omega_{\mu \mathrm{W}} \tau_{\mathrm{C}} \ll 1$, equivalent to $\tau_{1} \sim \tau_{2}$ [12]) being the most appropriate in our case. In a semilogarithmic plot (Fig. 4(a)) the linewidths linearly decrease as $T^{-1 / 4}$, suggesting a variable-range hopping [13] of the unpaired electrons. The characteristic correlation time $\tau_{\mathrm{C}}$ would be [13]

$1 / \tau_{\mathrm{C}}=\omega_{\mathrm{Hop}} \approx \omega_{0} \exp \left(-\frac{B}{T^{1 / 4}}\right)$

with $B \approx 1.66\left[\Lambda^{3} /\left(k_{\mathrm{B}} N\left(E_{\mathrm{F}}\right)\right)\right]^{1 / 4}$ and the decay length $\Lambda$ is assumed to not change with temperature, while the temperature dependence of the hopping range is $R \approx[0.83 \Lambda]^{1 / 4} /\left[N\left(E_{\mathrm{F}}\right) k_{\mathrm{B}} T\right]^{1 / 4}$ [13]. However, by substituting Eq. (3) into Eq. (2) and fitting our linewidths (Fig. 4(a)) the calculated hopping ranges are $R(295 \mathrm{~K})=0.08$ and $0.2 \AA$ or $R(4.3 \mathrm{~K})=0.3$ and $1.0 \AA$, (before and after annealing, respectively). They are unphysical and inconsistent with the inter-spin distances involved by the spin densities (>10 A, Section 3).

More realistic parameters could be obtained assuming the unpaired electrons to sit on the bandtails rather than at Fermi level [14], but the band 

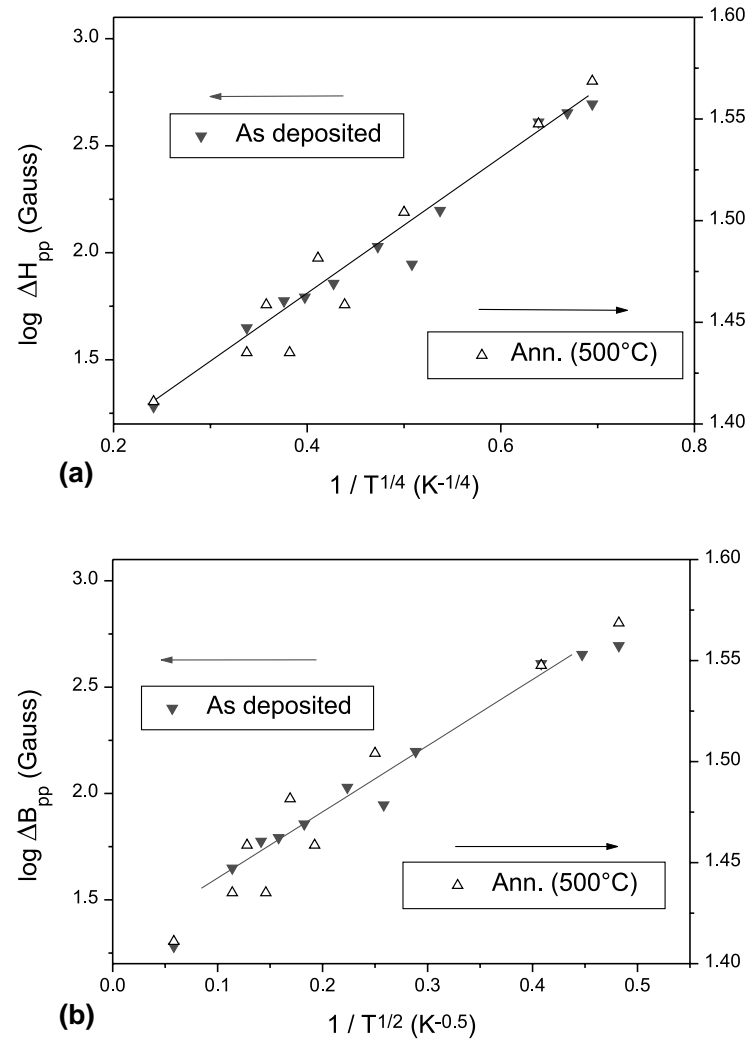

Fig. 4. The same data of Fig. 1 on a semilogarithmic plot (a) as a function of $T^{-1 / 4}$ (hinting a variable range hopping process) or (b) as a function of $T^{-1 / 2}$ (hinting a modified nearest neighbour hopping process, see text).

tails studied by photothermal deflection spectroscopy (PDS) on the same samples [15] $\left(<10^{18}\right.$ $\left.\mathrm{cm}^{3} \mathrm{eV}^{-1}\right)$ seem to rule out such hypothesis.

\subsection{Hopping in a non-diluted system with $\tau_{1} \sim \tau_{2}$}

A model using Eq. (2) was also recently proposed by Yokomichi and Morigaki (YM) [16] for fluorinated amorphous carbons (a-C:F, diluted systems exhibiting $N_{\mathrm{s}} \sim 10^{19} \mathrm{~cm}^{-3}$ and linewidths varying at low-temperature [10]). In a-C:F, YM found nearest neighbour hopping to be a realistic mechanism and use, instead of Eq. (3),

$$
\begin{aligned}
1 / \tau_{\mathrm{C}} & =\omega_{\text {Hop }} \\
& \approx \omega_{0} \exp \left(-\frac{2 R}{\Lambda}\right) \exp \left(-\frac{W_{0}}{k_{\mathrm{B}} T}\right) .
\end{aligned}
$$

Replacing Eq. (4) into Eq. (2), YM successfully explain the temperature dependence of the linewidths in a-C:F. The model applies indifferently in the cases of floating-bonds or electron hopping; however, in our ta-C case, the semilogarithmic trend of the linewidths versus $T^{-1}$ does not present any linear region, suggesting the YM model to need some modification.

Actually, Eqs. (1) and (3) are valid only under the hypothesis of site-to-site hops (i.e. diluted systems and $\left.\tau_{1} \ll \tau_{2}\right)$. In the presence of fast spinlattice processes $\left(\tau_{1} \sim \tau_{2}\right)$ the phonon population activated by the relaxing spins are so relevant that more than two sites are involved. Namely:

(1) The changes in distance $R \pm \delta R(T)$ between two contiguous spin packets due to the lattice vibrations are relevant. Let us consider that the energy associated to a phonon excited by a 'direct' spin-lattice process (see above) is approximately $\frac{1}{2} \mathrm{M} \omega_{0}^{2} \cdot \delta R(T)^{2} \sim k_{\mathrm{B}} T$, where $\omega_{0} \sim$ $\omega_{\mu \mathrm{W}} \sim 10 \mathrm{GHz}$ and $\mathrm{M}$ is the carbon ion mass. One ends up that, at room temperature, $\delta R \sim$ $0.18 R$ (as grown) and $\delta R \sim 0.12 R$ (after annealing). Since the hopping rate exponentially depends on $R$, it is clear that the effect must be considered. An $\exp \left[R / \Lambda-\left(k_{\mathrm{B}} / \mathrm{M}\right)^{1 / 2} /\left(\omega_{0} \cdot \Lambda\right)\right.$. $\left.T^{1 / 2}\right]$ dependence into Eq. (3) arises.

(2) In order to adapt Eq. (3) at the case of hops involving more than two sites, one must consider, at each temperature, the $n$ sites involved by the hop as a single, $n$-electron 'cluster' of size $\Lambda$. Indeed the 'bottlenecks' of the process will now become the 'cluster'-to-'cluster' hops. Curiously, a similar mechanism of inter-'cluster' hopping was already proposed to explain the dc conductivity of amorphous carbons [17]. The size $\Lambda$ of each $n$-electron 'cluster' increases with temperature as the hopping frequency increases, so that it needs to be optimised following the usual procedure (described for instance on Ref. [13]; i.e. minimising $\tau_{\mathrm{C}}$ into Eq. (3)). This implies a temperature dependence also for the size: $\Lambda=2 \cdot\left(k_{\mathrm{B}} / \mathrm{M}\right)^{1 / 2} \cdot T^{1 / 2} / \omega_{0}$, at $W_{0} \approx 0$.

As a consequence of points (1) and (2) Eq. (4), involved by the YM model, gives 
$1 / \tau_{\mathrm{C}}=\omega_{\text {Hop }} \approx \omega_{0} \exp \left[1-\frac{R \omega_{0}}{\left(k_{\mathrm{B}} / \mathrm{M}\right)^{1 / 2} T^{1 / 2}}\right]$.

Also Eq. (5), such as Eq. (3), could be successfully combined with Eq. (2) to give the correct trend of $\Delta H_{\mathrm{pp}}(\mathrm{T})$, that is strictly linear if plotted as a function of $T^{-1 / 2}$ (Fig. 4(b)). The parameters present into Eq. (5) (inter-spin distance $R$ and phonon frequency $\omega_{0} \sim \omega_{\mu \mathrm{W}} \sim 10 \mathrm{GHz}$ ) are all experimentally determinable (Section 3 ) providing slopes and intercepts which are correct with an accuracy of $\pm 20 \%$.

\section{Conclusions}

In conclusion, the nature of the hopping process responsible the temperature dependence of the linewidth in ta-C is unclear and will be the subject of further work supported by low-temperature dc conductivity and high frequency ESR measurements. The quasi-linear semilogarithmic trend vs. $T^{-1 / 4}$ seems to indicate a variable range hopping (VRH) mechanism. The difficulty to obtain realistic VRH parameters and the similarities with a-C:F rather than to amorphous silicon lead to suppose that nearest neighbour hopping is preferable. We have also shown the relevant role of annealing in reducing the temperature dependence of the linewidth.

\section{Acknowledgements}

D.D. acknowledges ICTP funding for Training and Research in Italian Laboratories. A.C.F. ac- knowledges funding from an EU Marie Curie TMR Fellowship. K.B.K.T. acknowledges support of the Association of Commonwealth Universities and the British Council.

\section{References}

[1] J. Robertson, Diam. Rel. Mater. 6 (1997) 212.

[2] H.J.V. Bardeleben, J.L. Cantin, A. Zeinert, B. Racine, K. Zellama, Appl. Phys. Lett. 78 (2001) 2843.

[3] T. Frauenheim, P. Blaudeck, U. Stephan, G. Jungnickel, Phys. Rev. B 50 (1994) 6709.

[4] F. Demichelis, C. Demartino, A. Tagliaferro, M. Fanciulli, Diam. Rel. Mater. 3 (1994) 844.

[5] H. Dersch, J. Stuke, J. Beichler, Phys. Status Solidi B 107 (1981) 307.

[6] A.C. Ferrari, A. Libassi, B.K. Tanner, V. Stolojan, J. Yuan, L.M. Brown, S.E. Rodil, B. Kleinsorge, J. Robertson, Phys. Rev. B 62 (2000) 11089.

[7] A. Abragam, B. Bleaney, Electron Paramagnetic Resonance of Transition Ions, Dover, New York, 1986.

[8] R.C. Barklie, M. Collins, S.R.P. Silva, Phys. Rev. B 61 (2000) 3546

[9] M. Hoinkis, E.D. Tober, R.L. White, M.S. Crowder, Appl. Phys. Lett. 61 (1992) 2653.

[10] N.H. Nickel, E.A. Schiff, Phys. Rev. B 58 (1998) 1114.

[11] U. Voget-Grote, J. Stuke, H. Wagner, in: G. Lucovsky, S. Galeener (Eds.), Proceedings of 1st International Conference on Structure and Excitation of Amorphous Solids, AIP Proceedings, vol. 31, 1976, p. 91.

[12] C.P. Slichter, Principles of Magnetic Resonance, 3rd Ed., Springer, Berlin, 1990.

[13] N.F. Mott, E.A. Davis, Electronic Processes in NonCrystalline Materials, Clarendon, Oxford, 1979.

[14] K.B.K. Teo, A.C. Ferrari, J. Robertson, W.I. Milne, G. Fanchini, A. Tagliaferro, E. Laurenti, Diam. Rel. Mater. (in press).

[15] C. Godet, these Proceedings, p. 333.

[16] H. Yokomichi, K. Morigaki, J. Non-Cryst. Solids 266-269 (2000) 797.

[17] D. Dasgupta, F. Demichelis, A. Tagliaferro, Philos. Mag. B 63 (1991) 1255. 